\title{
ANÁLISE DE VARIÁVEIS UTILIZADAS PARA A CLASSIFICAÇÃO DA GRAVIDADE DO DESVIO FONOLÓGICO
}

\author{
Analysis of variables used to classify the severity \\ of phonological disorders
}

\author{
Ana Rita Brancalioni ${ }^{(1)}$, Karine Faverzani Magnago ${ }^{(2)}$, Márcia Keske-Soares ${ }^{(3)}$
}

\begin{abstract}
RESUMO
Objetivo: analisar o comportamento das variáveis Percurso das Rotas, Nível de Complexidade e Aquisição de fonemas, conforme a classificação da gravidade do Desvio Fonológico (DF) por meio do Modelo Linguístico Fuzzy (BRANCALIONI, 2010). Além disso, verificar se a classificação da gravidade, por meio do Modelo, é capaz de diferenciar quantitativamente os graus quanto às classes de sons e aos traços distintivos. Método: a amostra foi constituída pela avaliação fonológica pré-tratamento de 204 sujeitos com DF, de ambos os sexos e idade entre quatro anos e dois meses a oito anos e dois meses. Todos os sujeitos foram submetidos à avaliação fonológica, avaliação da linguagem compreensiva e expressiva, avaliação do sistema estomatognático, exame articulatório repetitivo e avaliação audiológica. Foram realizadas as análises contrastiva e por traços distintivos. A gravidade dos 204 sistemas fonológicos desviantes foi classificada de acordo com o Modelo. Os valores correspondentes as variáveis foram tabulados e submetidos a tratamento estatístico por meio dos testes Tukey e Associação Qui-Quadrado complementado pela Análise de Resíduos Ajustados, considerando nível de significância de $5 \%(p<0,05)$. Resultados: verificou-se que as variáveis Percurso das Rotas, Nível de Complexidade e Aquisição de Fonemas diminuem conforme se acentua a gravidade, sendo estas relações estatisticamente significantes. Além disso, observou-se que o número de classe de som alterada e de substituições de traços distintivos aumenta conforme se acentua a gravidade, sendo estas relações estatisticamente significantes. Conclusão: o Modelo é capaz de diferenciar os graus quanto às variáveis, trazendo informações importantes de acordo com os diferentes graus.
\end{abstract}

DESCRITORES: Fala; Distúrbios da Fala; Índice de Gravidade de Doença; Classificação; Linguística

(1) Fonoaudióloga; Mestre em Distúrbios da Comunicação Humana pela Universidade Federal de Santa Maria UFSM.

(2) Bacharelado em Matemática; Professor Adjunto do Curso de Matemática e Pós-Graduação em Educação Matemática da Universidade Federal de Santa Maria - UFSM; Doutora em Matemática Aplicada pela Universidade Estadual de Campinas - UNICAMP, Campinas, SP, Brasil.

(3) Fonoaudióloga; Professor Associado do Curso de Fonoaudiologia e do Programa de Pós-graduação em Distúrbios da Comunicação Humana da Universidade Federal de Santa Maria - UFSM, Santa Maria, RS, Brasil; Bolsista CNPq - Brasil; Doutora em Linguística Aplicada pela Pontifícia Universidade Católica do Rio Grande do Sul - PUCRS, Porto Alegre, RS, Brasil.

Trabalho Realizado no Centro de Estudos de Linguagem e Fala (CELF) da Universidade Federal de Santa Maria UFSM.

Conflito de interesses: inexistente

\section{INTRODUÇÃO}

De acordo com a literatura ${ }^{1,2}$ em torno dos cinco anos a criança deve fazer uso adequado dos sons de sua língua materna nas diferentes posições silábicas. Entretanto, há crianças, que além dessa idade, apresentam substituições e/ou apagamentos dos sons da fala durante o processo de aquisição da linguagem ${ }^{3}$. Tais crianças não apresentam fatores orgânicos identificáveis, como deficiência auditiva, anormalidades anatômicas e funcionais, que interferem em sua fala, o que define o desvio fonológico ${ }^{4-7}$. Logo o desvio ocorre no nível fonológico e não no nível de produção mecânica dos sons $^{7}$. O desvio fonológico é comum na infância, 
sendo caracterizado por erros inapropriados de fala e redução da inteligibilidade ${ }^{8,9}$.

A gravidade do desvio fonológico é definida como a mensuração do quanto a capacidade de comunicação é inferior ou alterada em relação ao padrão adulto ${ }^{10}$. A gravidade é um dos aspectos clínicos na terapia fonoaudiológica frequentemente negligenciado ${ }^{11,12}$ quando a mesma não é considerada no momento da intervenção, visto que ela pode contribuir para a escolha do modelo terapêutico mais adequado e para a eficácia do tratamento ${ }^{13}$.

Uma vez que o comprometimento do sistema fonológico de crianças com desvio fonológico apresenta-se em graus variados $5,11,14$, a avaliação de fala deve fornecer parâmetros que auxiliem no estabelecimento da gravidade ${ }^{15}$ a fim de caracterizar diferentes sistemas fonológicos

Diversos estudos ${ }^{5,11,16-18}$ apontam a importância em classificar a gravidade do desvio fonológico. Contudo ainda não surgiu um consenso a respeito de todos os aspectos que precisam ser avaliados. Segundo estudo ${ }^{11}$ isso requer considerações de três parâmetros de mensuração: quais fatores precisam ser avaliados; como os vários limites de categorias devem ser definidos; e como as diferenças de idade podem ser contabilizadas.

Existem diferentes propostas para classificar a gravidade do desvio fonológico. Tais propostas baseiam-se principalmente em aspectos da fonologia como traços distintivos, processos fonológicos, aquisição de contrastes ${ }^{16-18}$ e também por meio do percentual de consoantes corretas, em suas diferentes considerações quanto à contagem das distorções ${ }^{19}$. Além disso, há um Modelo Linguístico Fuzzy ${ }^{20}$ desenvolvido para classificar a gravidade do desvio fonológico, que foi elaborado a partir do Modelo Implicacional de Complexidade dos Traços (MICT).

No Modelo Linguístico Fuzzy ${ }^{20}$ foram utilizadas como forma de análises as variáveis: Percurso das Rotas, Nível de Complexidade e Aquisição de fonemas. Visto que no Modelo Linguístico Fuzzy20 as variáveis foram analisadas simultaneamente, por meio de regras fuzzy, que se relacionam em paralelo, o objetivo deste trabalho é analisar o comportamento dessas variáveis, isoladamente, conforme a classificação da gravidade do Desvio Fonológico (DF) por meio do Modelo Linguístico Fuzzy ${ }^{20}$. Além disso, verificar se a classificação da gravidade por meio do Modelo Linguístico Fuzzy20 é capaz de diferenciar quantitativamente os graus quanto às classes de sons e aos traços distintivos. A importância desse trabalho se deve a melhor compreensão sobre o desvio fonológico em aspectos que podem diferenciá-los segundo a gravidade.

\section{MÉTODO}

Esta pesquisa caracteriza-se por ser de caráter transversal e do tipo quantitativo. A amostra foi constituída pela avaliação fonológica pré-tratamento de 204 sujeitos com desvio fonológico, na faixa etária de quatro anos e dois meses a oito anos e dois meses, sendo $73(35,8 \%)$ do sexo feminino e $131(64,2 \%)$ do sexo masculino.

Para compor a amostra desta pesquisa foram analisadas as avaliações fonoaudiológicas e os relatórios clínicos de 222 sujeitos, com desvio fonológico, que integram o banco de dados do Centro de Estudos de Linguagem e Fala de Instituição de Ensino superior. Além disso, 45 sujeitos com desvio fonológico, que aguardavam na lista de espera por tratamento fonoaudiológico, na clínica escola, foram recrutados para participar da pesquisa.

Todos os sujeitos foram submetidos à Avaliação Fonológica da Criança - AFC ${ }^{21}$, avaliação da linguagem compreensiva e expressiva, avaliação do sistema estomatognático, exame articulatório repetitivo e avaliação audiológica.

Como critério de inclusão os sujeitos deveriam apresentar, no sistema fonológico geral, no mínimo um fonema não adquirido ou parcialmente adquirido na posição de onset simples. Além disso, demonstrar compreensão adequada a sua idade mental, capacidade intelectual apropriada para o desenvolvimento da linguagem, e limiares audiológicos dentro do padrão de normalidade. Ainda, não apresentar anormalidade anatômica ou fisiológica do mecanismo de produção da fala e/ou disfunção neurológica e ser membro de uma família de falantes monolíngues do Português Brasileiro.

Os critérios de exclusão foram: alteração auditiva para a fala; sinais indicativos de Transtorno do Déficit de Atenção e Hiperatividade (TDAH), de síndromes ou de déficit cognitivo; ceceio anterior e/ ou lateral; comprometimento orgânico dos órgãos fonoarticulatórios (como por exemplo, fissura labiopalatina, frênulo lingual curto) ter realizado terapia fonoaudiológica anteriormente; idade inferior a 4 anos e dois meses e superior a oito anos e onze meses.

A idade mínima de quatro anos e dois meses justifica-se pela idade de aquisição do fonema /r/ na posição de onset medial para falantes do Português Brasileiro1. Já o limite de nove anos, caracteriza o período máximo do desenvolvimento da aquisição dos sons da fala, a partir dessa idade algumas crianças mantêm certos erros, tipicamente distorções dos sons da fala, caracterizando não um desvio ou atraso, mas erros residuais de fala ${ }^{22}$. 
O corpus de fala obtido por meio da nomeação espontânea, pela aplicação do instrumento Avaliação Fonológica da Criança ${ }^{21}$, foi gravado e armazenado em banco de dados. Posteriormente, essa coleta de fala foi transcrita foneticamente e realizada a análise contrastiva, cujo objetivo foi estabelecer o inventário fonético e o sistema fonológico da criança, para comparar com o alvo adulto. Também foi realizada análise por traços distintivos na qual foi adotado percentual de realizações corretas superior a $85 \%$ como critério de estabelecimento do traço e substituições de alta frequência percentagem acima de $15 \%^{16,23}$.

Para análise da condição de cada fonema considerou-se fonema presente quando se apresentou adquirido ou parcialmente adquirido e, fonema ausente quando se apresentou não adquirido ${ }^{20}$. Já para análise da classe de som considerou-se alterada quando um ou mais dos fonemas que compõem cada classe apresentaram-se não adquirido(s) no sistema fonológico.

A gravidade dos 204 sistemas fonológicos desviantes foi classificada de acordo com o Modelo Linguístico Fuzzy20 em Desvio Grave, ModeradoGrave, Moderado-Leve e Leve. Os valores correspondentes as variáveis Percurso das Rotas, Nível de Complexidade, Aquisição de Fonemas, classe de sons e traços distintivos foras tabulados em planilha eletrônica (Excel).

Os dados pertencem ao projeto: "Estudo dos desvios fonológicos: classificações e avaliações", aprovado pelo Comitê em Ética e Pesquisa de Instituição de Ensino Superior em seus aspectos éticos e metodológicos, sob número 23081.006440/2009-60 e Certificado de Apresentação para Apreciação Ética - CAAE número: 0093.0.243.000-09. O Termo de Consentimento Livre e Esclarecido (TCLE) foi assinado pelos pais e/ou responsáveis de todos os sujeitos participantes da pesquisa.

A fim de verificar se o Modelo Linguístico Fuzzy ${ }^{20}$ é capaz de diferenciar quantitativamente os graus quanto às variáveis foram utilizados o teste de Tukey e o Teste de Associação Qui-Quadrado complementado pela Análise de Resíduos Ajustados. Os testes estatísticos foram realizados no Software Bioestat 5.0 (2007) e o nível de significância foi de $5 \%(p<0,05)$.

\section{RESULTADOS}

Na Tabela 1 é apresentada a comparação da gravidade do desvio fonológico obtida pelo Modelo Linguístico Fuzzy ${ }^{20}$ com o Percurso das Rotas. Observa-se que a média do Percurso das Rotas (número de rotas percorridas) difere estatisticamente entre todos os graus. Além disso, o desvio Leve apresenta, em média, maior percurso (maior número de rotas percorridas), seguido do desvio Moderado-Leve, enquanto o desvio grave apresenta, em média, menor percurso (menor número de rotas percorridas), seguido do desvio Moderado-Grave.

Tabela 1 - Comparação da gravidade do desvio fonológico quanto ao número de Rotas Percorridas

\begin{tabular}{cc}
\hline \multirow{2}{*}{ Gravidade } & Percurso das Rotas \\
\cline { 2 - 2 } & Média \\
\hline DL & $14,58^{\mathrm{a}}$ \\
DML & $12,34^{\mathrm{b}}$ \\
DMG & $9,69^{\mathrm{c}}$ \\
DG & $5,05^{\mathrm{d}}$ \\
\hline
\end{tabular}

Legenda: $\mathrm{DL}=$ Desvio Leve; $\mathrm{DML}=$ Desvio Moderado Leve; $\mathrm{DMG}=$ Desvio Moderado Grave; $\mathrm{DG}=$ Desvio Grave; *letras iguais não diferem estatisticamente; ${ }^{* *}$ letras diferentes diferem estatisticamente

Nota: Análise estatística Teste de Tukey $(p<0,05)$

Na Figura 1 é ilustrada a distribuição da amostra quanto às Rotas Percorridas nos diferentes graus do desvio fonológico. Verifica-se associação estatisticamente significante entre as rotas e a gravidade do desvio, exceto para as rotas B2 e D1 que o número de sujeitos não foi suficiente para efetuar o teste estatístico.

Além disso, observam-se associações positivas para as rotas A1, B1, B4 e D2 estarem percorridas no grau Leve e não percorrida no grau Grave, mostrando que essas rotas são, frequentemente, mais percorridas no desvio Leve que no desvio Grave. Ainda, as associações observadas para as rotas A2, A3, B3, B5, B6, C1, C2, C3, C4 e D3 mostram que no Desvio Leve tais rotas são, em geral, percorridas, enquanto que no desvio Grave e Moderado-Grave essas rotas não são percorridas. 


\begin{tabular}{|c|c|c|c|c|c|c|c|}
\hline \multirow{3}{*}{\multicolumn{2}{|c|}{ ROTAS }} & \multicolumn{5}{|c|}{ GRAVIDADE } & \multirow{5}{*}{$\begin{array}{r}\begin{array}{r}\text { Valor } \\
\text { de } p\end{array} \\
0,004\end{array}$} \\
\hline & & \multirow{4}{*}{$\begin{array}{c}\text { DL }(\mathbf{n}=96) \\
\mathbf{n}(\%) \\
90(93,8)^{(+)} \\
6(6,2)^{(-)}\end{array}$} & \multirow{4}{*}{$\begin{array}{c}\text { DML (n=62) } \\
\mathbf{n}(\%) \\
54(87,1) \\
8(12,9)\end{array}$} & \multirow{4}{*}{$\begin{array}{c}\text { DMG }(\mathbf{n = 2 6 )} \\
\mathbf{n}(\%) \\
22(84,6) \\
4(15,4)\end{array}$} & \multirow{4}{*}{$\begin{array}{c}\text { DG (n=20) } \\
\mathbf{n}(\%) \\
13(65,0)^{(-)} \\
7(35,0)^{(+)}\end{array}$} & \multirow{4}{*}{$\begin{array}{c}\text { TOTAL } \\
n(\%) \\
179(87,7) \\
25(12,3)\end{array}$} & \\
\hline & & & & & & & \\
\hline \multirow{2}{*}{ A1 } & Percorrida & & & & & & \\
\hline & Não percorrida & & & & & & \\
\hline \multirow{2}{*}{ A2 } & Percorrida & $90(93,8)^{(+)}$ & $42(67,7)$ & $13(50,0)^{(-)}$ & $8(40,0)^{(-)}$ & $153(75,0)$ & \multirow{2}{*}{$<0,001$} \\
\hline & Não percorrida & $6(6,2)^{(-)}$ & $20(32,3)$ & $13(50,0)^{(+)}$ & $12(60,0)^{(+)}$ & $51(25,0)$ & \\
\hline \multirow{2}{*}{ A3 } & Percorrida & $95(98,7)^{(+)}$ & $53(85,5)$ & $18(69,2)^{(-)}$ & $4(20,0)(-)$ & $170(83,3)$ & \multirow{2}{*}{$<0,001$} \\
\hline & Não percorrida & $1(1,04)^{(-)}$ & $9(14,5)$ & $8(30,8)^{(+)}$ & $16(80,0)^{(+)}$ & $34(16,7)$ & \\
\hline \multirow{2}{*}{ B1 } & Percorrida & $96(100,0)^{(+)}$ & $55(88,7)$ & $21(80,8)$ & $10(50,0)^{(-)}$ & $182(89,2)$ & \multirow{2}{*}{$<0,001$} \\
\hline & Não percorrida & $0(0,0)^{(-)}$ & $7(11,3)$ & $5(19,2)$ & $10(50,0)^{(+)}$ & $22(10,8)$ & \\
\hline \multirow{2}{*}{ B2 } & Percorrida & $96(100,0)^{(+)}$ & $62(100,0)$ & $26(100,0)$ & $17(85,0)^{(-)}$ & $201(98,5)$ & \multirow{2}{*}{ \# } \\
\hline & Não percorrida & $0(0,0)^{(-)}$ & $0(0,00)$ & $0(0,0)$ & $3(15,0)^{(+)}$ & $3(1,5)$ & \\
\hline \multirow{2}{*}{ B3 } & Percorrida & $93(96,9)^{(+)}$ & $53(85,5)$ & $17(65,4)^{(-)}$ & $4(15,0)^{(-)}$ & $167(81,9)$ & \multirow{2}{*}{$<0,001$} \\
\hline & Não percorrida & $3(3,1)^{(-)}$ & $9(14,5)$ & $9(34,6)^{(+)}$ & $16(85,0)^{(+)}$ & $37(18,1)$ & \\
\hline \multirow{2}{*}{ B4 } & Percorrida & $88(91,7)^{(+)}$ & $46(74,2)$ & $17(65,4)$ & $7(35,0)^{(-)}$ & $158(77,5)$ & \multirow{2}{*}{$<0,001$} \\
\hline & Não percorrida & $8(8,3)^{(-)}$ & $16(25,8)$ & $9(34,6)$ & $13(65,0)^{(+)}$ & $46(22,5)$ & \\
\hline \multirow{2}{*}{ B5 } & Percorrida & $57(59,4)^{(+)}$ & $27(43,5)$ & $4(15,4)^{(-)}$ & $0(0,0)^{(-)}$ & $88(43,1)$ & \multirow{2}{*}{$<0,001$} \\
\hline & Não percorrida & $39(40,6)^{(-)}$ & $35(56,5)$ & $22(84,6)^{(+)}$ & $20(100,0)^{(+)}$ & $116(56,9)$ & \\
\hline \multirow{2}{*}{ B6 } & Percorrida & $95(99,0)^{(+)}$ & $53(85,5)$ & $18(69,2)^{(-)}$ & $4(20,0)^{(-)}$ & $170(83,3)$ & \multirow{2}{*}{$<0,001$} \\
\hline & Não percorrida & $1(1,0)^{(-)}$ & $9(14,5)$ & $8(30,8)^{(+)}$ & $16(80,0)^{(+)}$ & $34(16,7)$ & \\
\hline \multirow{2}{*}{$\mathrm{C} 1$} & Percorrida & $93(96,9)^{(+)}$ & $53(85,5)$ & $17(65,4)^{(-)}$ & $4(20,0)^{(-)}$ & $167(81,9)$ & \multirow{2}{*}{$<0,001$} \\
\hline & Não percorrida & $3(3,1)^{(-)}$ & $9(14,5)$ & $9(34,6)^{(+)}$ & $16(80,0)^{(+)}$ & $37(18,1)$ & \\
\hline \multirow{2}{*}{ C2 } & Percorrida & $95(99,0)^{(+)}$ & $53(85,5)$ & $18(69,2)^{(-)}$ & $4(20,0)^{(-)}$ & $170(83,3)$ & \\
\hline & Não percorrida & $1(1,0)^{(-)}$ & $9(14,5)$ & $8(30,8)^{(+)}$ & $16(80,0)^{(+)}$ & $34(16,7)$ & $<0,001$ \\
\hline & Percorrida & $87(90,6)^{(+)}$ & $42(67,7)$ & $7(26,9)^{(-)}$ & $1(5,0)^{(-)}$ & $137(67,2)$ & \\
\hline C3 & Não percorrida & $9(9,4)^{(-)}$ & $20(32,3)$ & $19(73,1)^{(+)}$ & $19(95,0)^{(+)}$ & $67(32,8)$ & $<0,001$ \\
\hline & Percorrida & $57(59,4)^{(+)}$ & $27(43,5)$ & $4(15,4)^{(-)}$ & $0(0,0)^{(-)}$ & $88(43,1)$ & \\
\hline C4 & Não percorrida & $39(40,6)^{(-)}$ & $35(56,5)$ & $22(84,6)^{(+)}$ & $20(100,0)^{(+)}$ & $116(56,9)$ & $<0,001$ \\
\hline D1 & Percorrida & $96(100,0)$ & $61(98,4)$ & $26(100,0)$ & $19(95,0)$ & $202(99,0)$ & \\
\hline D1 & Não percorrida & $0(0,0)$ & $1(1,6)$ & $0(0,0)$ & $1(5,0)$ & $2(1,0)$ & \# \\
\hline & Percorrida & $88(91,7)^{(+)}$ & $46(74,2)$ & $17(65,4)$ & $7(35,0)^{(-)}$ & $158(77,5)$ & \\
\hline D2 & Não percorrida & $8(8,3)^{(-)}$ & $16(25,8)$ & $9(34,6)$ & $13(65,0)^{(+)}$ & $46(22,5)$ & $<0,001$ \\
\hline & Percorrida & $87(90,6)^{(+)}$ & $42(67,7)$ & $7(26,9)^{(-)}$ & $1(5,0)^{(-)}$ & $137(67,2)$ & \\
\hline D3 & Não percorrida & $9(9,4)^{(-)}$ & $20(32,3)$ & $19(73,1)^{(+)}$ & $19(95,0)^{(+)}$ & $67(32,8)$ & $<0,001$ \\
\hline
\end{tabular}

Legenda: $\mathrm{DL}=$ Desvio Leve; $\mathrm{DML}=$ Desvio Moderado Leve; $\mathrm{DMG}=$ Desvio Moderado Grave; $\mathrm{DG}=\mathrm{Desvio}$ Grave.

Nota: valor de $p=$ significância do teste de Associação Qui-Quadrado; \#: número não suficiente para efetuar o teste estatístico. Análise de resíduos: (+) Associação significante positiva (-) Associação significante negativa

Figura 1 - Análise das rotas percorridas conforme a gravidade do Desvio Fonológico

Na Tabela 2 é apresentada a comparação da gravidade do desvio fonológico com o Nível de Complexidade. Observa-se que a média obtida para a variável Nível de Complexidade difere estatisticamente entre todos os graus. Além disso, o desvio Leve alcança, em média, nível de complexidade mais elevado, seguido do desvio Moderado-Leve, enquanto o desvio grave alcança, em média, nível de complexidade mais baixos, seguido do desvio Moderado-Grave.
Tabela 2 - Comparação da gravidade do desvio fonológico quanto ao Nível de Complexidade

\begin{tabular}{cc}
\hline \multirow{2}{*}{ Gravidade } & Nível de Complexidade \\
\cline { 2 - 2 } & Média \\
\hline DL & $8,52^{\mathrm{a}}$ \\
DML & $8,11^{\mathrm{b}}$ \\
DMG & $7,31^{\mathrm{c}}$ \\
DG & $5,05^{\mathrm{d}}$ \\
\hline
\end{tabular}

Legenda: $\mathrm{DL}=$ Desvio Leve; $\mathrm{DML}=$ Desvio Moderado Leve; $\mathrm{DMG}=$ Desvio Moderado Grave; $\mathrm{DG}=$ Desvio Grave; *letras iguais não diferem estatisticamente; ${ }^{*}$ letras diferentes diferem estatisticamente

Nota: Análise estatística Teste de Tukey 


\begin{tabular}{|c|c|c|c|c|c|}
\hline \multirow{3}{*}{$\begin{array}{c}\text { Nível de } \\
\text { Complexidade }\end{array}$} & \multicolumn{4}{|c|}{ GRAVIDADE } & \multirow{3}{*}{ Valor de $p$} \\
\hline & \multirow{2}{*}{$\frac{\mathrm{DL}}{\mathrm{n}(\%)}$} & \multirow{2}{*}{$\begin{array}{l}\text { DML } \\
n(\%)\end{array}$} & \multirow{2}{*}{$\begin{array}{l}\text { DMG } \\
n(\%)\end{array}$} & \multirow{2}{*}{$\begin{array}{c}\text { DG } \\
\mathrm{n}(\%)\end{array}$} & \\
\hline & & & & & \\
\hline N1 ao N6 & $0(0,0)^{(-)}$ & $4(6,4)$ & $5(19,2)$ & $15(75,0)^{(+)}$ & \\
\hline N7 & $9(9,4)^{(-)}$ & $13(21,0)$ & $12(46,2)^{(+)}$ & $4(20,0)$ & \\
\hline N8 & $30(31,2)$ & $17(27,4)$ & $6(23,1)$ & $1(5,0)^{(-)}$ & $<0,001$ \\
\hline N9 & $57(59,4)^{(+)}$ & $28(45,2)$ & $3(11,5)^{(-)}$ & $0(0,0)^{(-)}$ & \\
\hline TOTAL & $96(100,0)$ & $62(100,0)$ & $26(100,0)$ & $20(100,0)$ & \\
\hline
\end{tabular}

Legenda: $\mathrm{DL}=$ Desvio Leve; $\mathrm{DML}=$ Desvio Moderado Leve; $\mathrm{DMG}=$ Desvio Moderado Grave; $\mathrm{DG}=\mathrm{Desvio}$ Grave. Nota: Valor de $p=$ significância do teste de Associação Qui-Quadrado;

Análise de resíduos: (+) Associação significante positiva (-) Associação significante negativa.

Figura 2 - Análise do Nível de Complexidade conforme a gravidade do Desvio Fonológico

A Figura 2 ilustra a distribuição da amostra conforme a variável Nível de Complexidade, para os diferentes graus do desvio fonológico. Verifica-se associação estatisticamente significante entre - Nível de Complexidade e a gravidade do desvio fonológico. Além disso, observa-se associação significante positiva entre o Nível 9 e o Desvio Leve; entre o Nível 7 e o Desvio Moderado-Grave; e entre os Níveis 1 a 6 e o Desvio Grave.

Na Tabela 3 é apresentada a comparação da gravidade do desvio fonológico com a Aquisição dos Fonemas. Verifica-se que a média obtida para a aquisição dos fonemas difere estatisticamente entre todos os graus. Além disso, o desvio Leve apresenta, em média, maior pontuação correspondente à Aquisição dos Fonemas, seguido do desvio Moderado-Leve, enquanto o desvio Grave apresenta, em média, menor pontuação, seguido do desvio Moderado-Grave

Tabela 3 - Comparação da gravidade do desvio fonológico quanto à Aquisição dos Fonemas

\begin{tabular}{cc}
\hline \multirow{2}{*}{ Gravidade } & Aquisição dos Fonemas \\
\cline { 2 - 2 } & Média \\
\hline DL & $17,18^{\mathrm{a}}$ \\
DML & $13,69^{\mathrm{b}}$ \\
DMG & $11,21^{\mathrm{c}}$ \\
DG & $7,95^{\mathrm{d}}$ \\
\hline
\end{tabular}

Legenda: DL= Desvio Leve; DML= Desvio Moderado Leve; $\mathrm{DMG}=$ Desvio Moderado Grave; $\mathrm{DG}=$ Desvio Grave *letras iguais não diferem estatisticamente; ${ }^{* *}$ letras diferentes diferem estatisticamente.

Nota: Análise estatística Teste de Tukey.
Na Figura 3 é ilustrada a distribuição da amostra conforme a presença/ausência de cada fonema, de acordo com a gravidade do desvio fonológico. Verificou-se associação estatisticamente significante entre os fonemas e a gravidade do desvio fonológico, exceto $/ \mathrm{m} /, / \mathrm{n} /, / \mathrm{n} /, / \mathrm{p} /$ e $/ \mathrm{t} /$, que o número de sujeitos não foi suficiente para efetuar o teste estatístico.

Além disso, verifica-se que as associações obtidas entre os fonemas / / /, /f/ e /R/ e a gravidade do desvio fonológico referem-se aos desvio Leve e Grave, revelando que esses fonemas estão mais frequentemente presentes no desvio Leve que no desvio Grave. Já para os fonemas /b/, /d/, /g/, /v/, /S/, /3/, /l/, / $\mathrm{N}$ e /r/ obteve-se associação entre o grau Leve e os graus Moderado-Grave e Grave. Tais achados mostram que esses fonemas estão em geral presentes no desvio Leve e ausentes no desvio Moderado-Grave e Grave.

Ainda, houve associação entre o fonema /z/ e a gravidade, sendo esta observada entre o grau Leve e os graus Moderado-Leve, Moderado-Grave e Grave. Por fim, verificou-se associação entre o fonema /s/ e a gravidade no grau Leve e nos graus Moderado-Leve e Grave.

$\mathrm{Na}$ Tabela 4 é apresentada a distribuição da gravidade do desvio fonológico conforme o número de classes de sons alteradas. No desvio Leve verifica-se associação significante positiva para a presença de nenhuma e de apenas uma classe de sons. No desvio Moderado-Leve verifica-se associação significante positiva para a presença de duas classes de sons. Nos desvios Moderado-Grave e Grave observa-se associação significante positiva para a presença de três ou quatro classes de sons. 


\begin{tabular}{|c|c|c|c|c|c|c|c|}
\hline \multirow{3}{*}{\multicolumn{2}{|c|}{ Fonemas }} & \multicolumn{5}{|c|}{ Gravidade } & \multirow{5}{*}{$\begin{array}{c}\begin{array}{c}\text { Valor } \\
\text { de p }\end{array} \\
\#\end{array}$} \\
\hline & & \multirow{3}{*}{$\begin{array}{c}\mathrm{DL}(\mathbf{n = 9 6 )} \\
\mathbf{n}(\%) \\
96(100,0)\end{array}$} & \multirow{3}{*}{$\begin{array}{c}\operatorname{DML}(\mathbf{n}=\mathbf{6 2}) \\
\mathbf{n}(\%) \\
62(100,0)\end{array}$} & \multirow{3}{*}{$\begin{array}{c}\text { DMG }(\mathbf{n}=26) \\
\mathbf{n}(\%) \\
26(100,0)\end{array}$} & \multirow{3}{*}{$\begin{array}{c}\text { DG (n=20) } \\
\mathbf{n}(\%) \\
20(100,0)\end{array}$} & \multirow{3}{*}{$\begin{array}{c}\text { TOTAL } \\
204(100,0)\end{array}$} & \\
\hline & & & & & & & \\
\hline \multirow{2}{*}{$/ \mathrm{m} /$} & Presente & & & & & & \\
\hline & Ausente & $0(0,0)$ & $0(0,0)$ & $0(0,0)$ & $0(0,0)$ & $0(0,0)$ & \\
\hline \multirow{2}{*}{$/ \mathrm{n} /$} & Presente & $96(100,0)$ & $62(100,0)$ & $26(100,0)$ & $18(90,0)$ & $202(99,0)$ & \multirow{2}{*}{ \# } \\
\hline & Ausente & $0(0,0)$ & $0(0,0)$ & $0(0,0)$ & $2(10,0)$ & $2(1,0)$ & \\
\hline \multirow{2}{*}{$/ \mathrm{n} /$} & Presente & $96(100,0)$ & $61(98,4)$ & $26(100,0)$ & $19(95,0)$ & $202(99,0)$ & \multirow{2}{*}{ \# } \\
\hline & Ausente & $0(0,0)$ & $1(1,6)$ & $0(100,0)$ & $1(5,0)$ & $2(1,0)$ & \\
\hline \multirow{2}{*}{$/ p /$} & Presente & $96(100,0)$ & $62(100,0)$ & $26(100,0)$ & $19(95,0)$ & $203(99,5)$ & \multirow{2}{*}{ \# } \\
\hline & Ausente & $0(0,0)$ & $0(0,0)$ & $0(100,0)$ & $1(5,0)$ & $1(0,5)$ & \\
\hline \multirow{2}{*}{$/ \mathrm{b} /$} & Presente & $96(100,0)^{(+)}$ & $51(82,3)$ & $9(34,6)^{(-)}$ & $11(55,0)^{(-)}$ & $167(81,9)$ & \multirow{2}{*}{$<0,001$} \\
\hline & Ausente & $0(0,0)^{(-)}$ & $11(17,7)$ & $17(65,4)^{(+)}$ & $9(45,0)^{(+)}$ & $37(18,1)$ & \\
\hline \multirow{2}{*}{$/ \mathrm{t} /$} & Presente & $96(100,0)$ & $60(96,8)$ & $25(96,2)$ & $17(85,0)$ & $198(97,0)$ & \multirow[b]{2}{*}{ \# } \\
\hline & Ausente & $0(0,0)$ & $2(3,2)$ & $1(3,8)$ & $3(15,0)$ & $6(3,0)$ & \\
\hline \multirow{2}{*}{$/ \mathrm{d} /$} & Presente & $93(96,9)^{(+)}$ & $52(83,9)$ & $16(61,5)^{(-)}$ & $9(45,0)^{(-)}$ & $170(83,3)$ & \multirow{2}{*}{$<0,001$} \\
\hline & Ausente & $3(3,1)^{(-)}$ & $10(16,1)$ & $10(38,5)^{(+)}$ & $11(55,0)^{(+)}$ & $34(16,7)$ & \\
\hline \multirow{2}{*}{$/ \mathbf{k} /$} & Presente & $90(93,8)^{(+)}$ & $53(85,5)$ & $22(84,6)$ & $13(65,0)^{(-)}$ & $178(87,3)$ & \multirow{2}{*}{0,005} \\
\hline & Ausente & $6(6,2)^{(-)}$ & $9(14,5)$ & $4(15,4)$ & $7(35,0)^{(+)}$ & $26(12,7)$ & \\
\hline \multirow{2}{*}{$/ g /$} & Presente & $90(93,8)^{(+)}$ & $43(69,4)$ & $13(50,0)^{(-)}$ & $6(30,0)^{(-)}$ & $152(74,5)$ & \multirow{2}{*}{$<0,001$} \\
\hline & Ausente & $6(6,2)^{(-)}$ & $19(30,6)$ & $13(50,0)^{(+)}$ & $14(70,0)^{(+)}$ & $52(25,5)$ & \\
\hline & Presente & $96(100,0)^{(+)}$ & $58(93,6)$ & $23(88,5)$ & $12(60,0)^{(-)}$ & $189(92,6)$ & \\
\hline$/ \mathrm{t} /$ & Ausente & $0(0,0)^{(-)}$ & $4(6,4)$ & $3(11,5)$ & $8(40,0)^{(+)}$ & $15(7,4)$ & $<0,001$ \\
\hline I & Presente & $95(99,0)^{(+)}$ & $51(82,3)$ & $16(61,5)^{(-)}$ & $9(45,0)^{(-)}$ & $171(83,8)$ & \\
\hline IVI & Ausente & $1(1,0)^{(-)}$ & $11(17,74)$ & $10(38,5)^{(+)}$ & $11(55,0)^{(+)}$ & $33(16,2)$ & $<0,001$ \\
\hline & Presente & $95(99,0)^{(+)}$ & $48(77,4)^{(-)}$ & $21(80,8)$ & $10(50,0)^{(-)}$ & $174(85,3)$ & \\
\hline /s/ & Ausente & $1(1,0)^{(-)}$ & $14(22,6)(+)$ & $5(19,2)$ & $10(50,0)^{(+)}$ & $30(14,7)$ & $<0,001$ \\
\hline & Presente & $95(99,0)^{(+)}$ & $40(64,5)^{(-)}$ & $14(53,9)^{(-)}$ & $7(35,0)^{(-)}$ & $156(76,5)$ & \\
\hline $\mid \mathbf{z I}$ & Ausente & $1(1,0)^{(-)}$ & $22(35,5)^{(+)}$ & $12(46,1)^{(+)}$ & $13(65,0)^{(+)}$ & $48(23,5)$ & J,001 \\
\hline & Presente & $87(90,6)^{(+)}$ & $42(67,7)$ & $14(53,9)^{(-)}$ & $7(35,0)^{(-)}$ & $150(73,5)$ & \\
\hline $13 /$ & Ausente & $9(9,4)^{(-)}$ & $20(32,3)$ & $12(46,1)^{(+)}$ & $13(65,0)^{(+)}$ & $54(26,5)$ & 0,001 \\
\hline & Presente & $81(84,4)^{(+)}$ & $32(51,6)$ & $7(26,9)^{(-)}$ & $4(20,0)^{(-)}$ & $124(60,8)$ & \\
\hline$|3|$ & Ausente & $15(15,6)^{(-)}$ & $30(48,4)$ & $19(73,1)^{(+)}$ & $16(80,0)^{(+)}$ & $80(39,2)$ & $<0,0$ \\
\hline & Presente & $94(97,9)^{(+)}$ & $54(87,1)$ & $17(65,4)^{(-)}$ & $3(15,0)^{(-)}$ & $168(82,4)$ & \\
\hline III & Ausente & $2(2,1)^{(-)}$ & $8(12,9)$ & $9(34,6)^{(+)}$ & $17(85,0)^{(+)}$ & $36(17,6)$ & \\
\hline & Presente & $87(90,6)^{(+)}$ & $42(67,7)$ & $7(26,9)^{(-)}$ & $1(5,0)^{(-)}$ & $137(67,2)$ & \\
\hline$I \lambda I$ & Ausente & $9(9,4)^{(-)}$ & $20(32,3)$ & $19(73,1)^{(+)}$ & $19(95,0)^{(+)}$ & $67(32,8)$ & 0,001 \\
\hline & Presente & $94(97,9)^{(+)}$ & $50(80,7)$ & $18(69,2)$ & $4(20,0)^{(-)}$ & $166(81,4)$ & \\
\hline /R/ & Ausente & $2(2,1)^{(-)}$ & $12(19,3)$ & $8(30,8)$ & $16(80,0)^{(+)}$ & $38(18,6)$ & $<0,001$ \\
\hline$|r|$ & Presente & $57(59,4)^{(+)}$ & $29(46,8)$ & $4(15,4)^{(-)}$ & $0(0,0)^{(-)}$ & $90(44,1)$ & \\
\hline Ir/ & Ausente & $39(40,6)^{(-)}$ & $33(53,2)$ & $22(84,6)^{(+)}$ & $20(100,0)^{(+)}$ & $114(55,9)$ & $<0,001$ \\
\hline
\end{tabular}

Legenda: $\mathrm{DL}=$ Desvio Leve; $\mathrm{DML}=$ Desvio Moderado Leve; $\mathrm{DMG}=$ Desvio Moderado Grave; $\mathrm{DG}=$ Desvio Grave.

Nota: Valor de $\mathrm{p}=$ significância do teste de Associação Qui-Quadrado; \#: número não suficiente para efetuar o teste estatístico.

Análise de resíduos: (+) Associação significante positiva (-) Associação significante negativa.

Figura 3 - Condição de cada fonema conforme a gravidade do desvio fonológico

Tabela 4 - Distribuição da gravidade do desvio fonológico quanto ao número de classes de sons alteradas

\begin{tabular}{lccccc}
\hline \multirow{2}{*}{$\begin{array}{l}\text { Número de Classe(s) } \\
\text { de Alterada(s) }\end{array}$} & $\mathbf{5}$ & $\mathbf{5}$ GRA & $\mathbf{D M G}$ & $\mathbf{D G}$ & \multirow{2}{*}{ Valor de $\mathbf{p}$} \\
\cline { 2 - 5 } & $\mathbf{n ~ ( \% )}$ & $\mathbf{n ~ ( \% )}$ & $\mathbf{n}(\%)$ & $\mathbf{n}(\%)$ & \\
\hline Nenhuma & $32(33,3)^{(+)}$ & $1(1,6)^{(-)}$ & $0(0,0)^{(-)}$ & $0(0,0)^{(-)}$ & \\
Uma & $60(62,5)^{(+)}$ & $14(22,6)^{(-)}$ & $1(3,8)^{(-)}$ & $0(0,0)^{(-)}$ & \multirow{2}{*}{$\mathbf{0 , 0 0 1}$} \\
Duas & $4(4,2)^{(-)}$ & $39(62,9)^{(+)}$ & $8(30,8)$ & $5(25,0)$ & \\
Três ou Quatro & $0(0,0)^{(-)}$ & $8(30,8)$ & $17(65,4)^{(+)}$ & $15(75,0)^{(+)}$ & \\
\hline TOTAL & $96(100,0)$ & $62(100,0)$ & $26(100,0)$ & $20(100,0)$ & \\
\hline
\end{tabular}

Legenda: $\mathrm{DL}=$ Desvio Leve; $\mathrm{DML}=$ Desvio Moderado Leve; $\mathrm{DMG}=$ Desvio Moderado Grave; $\mathrm{DG}=\mathrm{Desvio}$ Grave.

Nota: Valor de $p=$ significância do teste de Associação Qui-Quadrado. Análise de resíduos: (+) Associação significante positiva (-)

Associação significante negativa. 
Na Figura 4 é apresentada a distribuição entre a gravidade do desvio fonológico e o número de classes de sons alteradas. Verifica-se associação estatisticamente significante entre as classes das plosivas, das fricativas e das líquidas com a gravidade do desvio fonológico.
Observa-se que as plosivas e as fricativas estão frequentemente mais alteradas nos desvios Moderado-Leve, Moderado-Grave e Grave que no desvio Leve. Além disso, na classe das líquidas, todos os sujeitos classificados com Desvio Moderado-Grave e Grave apresentaram esta classe alterada, havendo associação significante positiva.

\begin{tabular}{|c|c|c|c|c|c|c|c|}
\hline \multirow{2}{*}{\multicolumn{2}{|c|}{ Classes de Sons }} & \multicolumn{5}{|c|}{ Gravidade } & \multirow{3}{*}{ Valor de $p$} \\
\hline & & \multirow{2}{*}{$\begin{array}{c}\text { DL } \\
n(\%) \\
\end{array}$} & \multirow{2}{*}{$\begin{array}{c}\text { DML } \\
\mathrm{n}(\%) \\
\end{array}$} & \multirow{2}{*}{$\begin{array}{c}\text { DMG } \\
\mathbf{n}(\%)\end{array}$} & \multirow{2}{*}{$\frac{D G}{n(\%)}$} & \multirow{2}{*}{ TOTAL } & \\
\hline & & & & & & & \\
\hline \multirow{2}{*}{ Nasais } & Não Alteradas & $96(100,0)$ & $61(98,4)$ & $26(100,0)$ & $17(85,0)$ & $200(98,0)$ & \multirow{2}{*}{$\#$} \\
\hline & Alteradas & $0(0,0)$ & $1(1,6)$ & $0(0,0)$ & $3(15,0)$ & $4(2,0)$ & \\
\hline \multirow{2}{*}{ Plosivas } & Não Alteradas & $87(90,6)^{(+)}$ & $34(54,8)^{(-)}$ & $9(34,6)^{(-)}$ & $3(15,0)^{(-)}$ & $133(65,2)$ & \multirow{2}{*}{$<0,001$} \\
\hline & Alteradas & $9(9,4)^{(-)}$ & $28(45,2)^{(+)}$ & $17(65,4)^{(+)}$ & $17(85,0)^{(+)}$ & $71(34,8)$ & \\
\hline \multirow{2}{*}{ Fricativas } & Não Alteradas & $78(81,3)^{(+)}$ & $15(24,2)^{(-)}$ & $1(3,8)^{(-)}$ & $2(10,0)^{(-)}$ & $96(47,1)$ & \multirow{2}{*}{$<0,001$} \\
\hline & Alteradas & $18(18,7)^{(-)}$ & $47(75,8)^{(+)}$ & $25(96,2)^{(+)}$ & $18(90,0)^{(+)}$ & $108(52,9)$ & \\
\hline \multirow{2}{*}{ Líquidas } & Não Alteradas & $56(58,3)^{(+)}$ & $22(35,5)$ & $0(0,0)^{(-)}$ & $0(0,0)^{(-)}$ & $78(38,2)$ & \multirow{2}{*}{$<0,001$} \\
\hline & Alteradas & $40(41,7)^{(-)}$ & $40(64,5)$ & $26(100,0)^{(+)}$ & $20(100,0)^{(+)}$ & $126(61,8)$ & \\
\hline
\end{tabular}

Legenda: DL - Desvio Leve; DML - Desvio Moderado Leve; DMG - Desvio Moderado Grave; DG - Desvio Grave.

Nota: Valor de $p=$ significância do teste de Associação Qui-Quadrado; \#: número não suficiente para efetuar o teste estatístico. Análise de resíduos: (+) Associação significante positiva (-) Associação significante negativa.

Figura 4 - Classes de Sons alteradas conforme a gravidade

A Tabela 5 apresenta a comparação entre a gravidade do desvio fonológico e a quantidade de substituições de traços distintivos. Verifica-se que a média do número de substituições de traços distintivos difere estatisticamente entre todos os graus. Também se observa que o desvio Leve apresenta, em média, menor quantidade de substituição de traços distintivos, seguido do desvio Moderado-Leve, enquanto que os desvios Grave e Moderado-Grave apresentam, em média, maior quantidade de substituição de traços distintivos.

Tabela 5 - Comparação da gravidade do desvio fonológico quanto à quantidade de substituições de traços distintivos

\begin{tabular}{cc}
\hline \multirow{2}{*}{ Gravidade } & $\begin{array}{c}\text { Substituições de Traços } \\
\text { Distintivos }\end{array}$ \\
\cline { 2 - 2 } & Média \\
\hline DL & $3,18^{\mathrm{a}}$ \\
DML & $7,50^{\mathrm{b}}$ \\
DMG & $11,81^{\mathrm{c}}$ \\
DG & $18,00^{\mathrm{d}}$ \\
\hline
\end{tabular}

Legenda: $\mathrm{DL}=$ Desvio Leve; $\mathrm{DML}=$ Desvio Moderado Leve; $\mathrm{DMG}=$ Desvio Moderado Grave; $\mathrm{DG}=$ Desvio Grave; *letras iguais não diferem estatisticamente; **letras diferentes diferem estatisticamente.

Nota: Análise estatística Teste de Tukey.
A Figura 5 ilustra as substituições dos traços distintivos conforme a gravidade do desvio fonológico. Verificou-se associação estatisticamente significante entre as substituições de traços distintivos e a gravidade do desvio, exceto para as seguintes: [+soan] $\rightarrow$ [-soan]; [-soan] $\rightarrow$ [+soan]; [-aprox] $\rightarrow$ [+aprox]; [+voc] $\rightarrow$ [-voc]; [-glot] $\rightarrow$ [+glot]; [-cont] $\rightarrow$ [+cont]; [lab] $\rightarrow$ [dors]; [cor] $\rightarrow$ [lab]; [dors] $\rightarrow$ [lab] e [cor] $\rightarrow$ [ant] $(+\rightarrow-)$.

\section{DISCUSSÃO}

Quanto à variável percurso das rotas os achados revelam que a gravidade do desvio aumenta com a diminuição do Percurso das Rotas (número de rotas percorridas), corroborando a afirmação de estudo ${ }^{7}$ que quanto menor o número de rotas percorridas maior é a gravidade do desvio fonológico. Além disso, os achados concordam com os dados de outros estudos ${ }^{16,18}$ que revelaram que os desvios mais graves apresentavam maior número de rotas não percorridas.

A partir da análise individual das rotas, segundo a gravidade, pode-se inferir que as rotas D1 e B2 são percorridas independente da gravidade, contudo quando não percorridas trata-se, em geral, de desvio Grave. Concordando com o estudo ${ }^{16} \mathrm{em}$ que todos os sujeitos com desvio fonológico de 


\begin{tabular}{|c|c|c|c|c|c|c|c|c|}
\hline \multirow{3}{*}{\multicolumn{3}{|c|}{$\begin{array}{l}\text { Substituições de Traços } \\
\text { Distintivos }\end{array}$}} & \multicolumn{5}{|c|}{ Gravidade } & \multirow{3}{*}{$\begin{array}{l}\text { Valor } \\
\text { de } p\end{array}$} \\
\hline & & & \multirow{2}{*}{$\begin{array}{c}\mathrm{DL} \\
\mathrm{n}(\%)\end{array}$} & \multirow{2}{*}{$\begin{array}{c}\text { DML } \\
\mathrm{n}(\%)\end{array}$} & \multirow{2}{*}{$\begin{array}{l}\text { DMG } \\
\mathrm{n}(\%)\end{array}$} & \multirow{2}{*}{$\begin{array}{c}\text { DG } \\
\mathrm{n}(\%)\end{array}$} & \multirow{2}{*}{ TOTAL } & \\
\hline & & & & & & & & \\
\hline \multirow{4}{*}{ Soan } & \multirow{2}{*}{$+\rightarrow-$} & Não ocorre & $94(97,9)$ & $60(96,8)$ & $21(80,8)$ & $17(85,0)$ & $192(94,1)$ & \multirow[b]{2}{*}{ \# } \\
\hline & & Ocorre & $2(2,1)$ & $2(3,2)$ & $5(19,2)$ & $3(15,0)$ & $12(5,9)$ & \\
\hline & \multirow{2}{*}{$-\rightarrow+$} & Não ocorre & $95(99,0)$ & $58(93,6)$ & $24(92,3)$ & $17(85,0)$ & $194(95,1)$ & \multirow{2}{*}{$\#$} \\
\hline & & Ocorre & $1(1,0)$ & $4(6,4)$ & $2(7,7)$ & $3(15,0)$ & $10(4,9)$ & \\
\hline \multirow{4}{*}{ Aprox } & \multirow{2}{*}{$+\rightarrow-$} & Não ocorre & $52(54,2)$ & $36(58,1)$ & $10(38,5)$ & $2(10,0)^{(-)}$ & $100(49,0)$ & \multirow{2}{*}{$<0,001$} \\
\hline & & Ocorre & $44(45,8)$ & $26(41,9)$ & $16(61,5)$ & $18(90,0)^{(+)}$ & $104(51,0)$ & \\
\hline & \multirow{2}{*}{$-\rightarrow+$} & Não ocorre & $96(100,0)$ & $60(96,8)$ & $26(100,0)$ & $17(85,0)$ & $199(97,6)$ & \multirow{2}{*}{$\#$} \\
\hline & & Ocorre & $0(0,0)$ & $2(3,2)$ & $0(0,0)$ & $3(15,0)$ & $5(2,4)$ & \\
\hline \multirow{4}{*}{ Voc } & \multirow{2}{*}{$+\rightarrow-$} & Não ocorre & $96(100,0)$ & $62(100,0)$ & $25(96,2)$ & $20(95,0)$ & $202(99,0)$ & \multirow{2}{*}{$\#$} \\
\hline & & Ocorre & $0(0,0)$ & $0(0,0)$ & $1(1,8)$ & $1(5,0)$ & $2(1,0)$ & \\
\hline & \multirow{2}{*}{$-\rightarrow+$} & Não ocorre & $54(56,2)$ & $37(59,7)$ & $13(50,0)$ & $3(15,0)^{(-)}$ & $107(52,5)$ & \multirow{2}{*}{0,004} \\
\hline & & Ocorre & $42(43,8)$ & $25(40,3)$ & $13(50,0)$ & $17(85,0)^{(+)}$ & $97(47,5)$ & \\
\hline \multirow{4}{*}{ Voz } & & Não Ocorre & $80(83,3)^{(+)}$ & $27(43,5)^{(-)}$ & $6(23,1)^{(-)}$ & $9(45,0)$ & $122(59,8)$ & 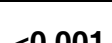 \\
\hline & $+7-$ & Ocorre & $16(16,7)^{(-)}$ & $35(56,5)^{(+)}$ & $20(76,9)^{(+)}$ & $11(55,0)$ & $82(40,2)$ & $<0,00 \mid$ \\
\hline & & Não ocorre & $94(97,9)$ & $58(93,6)$ & $23(88,5)$ & $19(95,0)$ & $194(95,1)$ & \\
\hline & & Ocorre & $2(2,1)$ & $4(6,4)$ & $3(11,5)$ & $1(5,0)$ & $10(4,9)$ & \# \\
\hline Glot & & Não ocorre & $96(100,0)$ & $62(100,0)$ & $25(96,2)$ & $19(95,0)$ & $202(99,0)$ & \\
\hline Cons & & Ocorre & $0(0,0)$ & $0(0,0)$ & $1(3,8)$ & $1(5,0)$ & $2(1,0)$ & \# \\
\hline & & Não ocorre & $57(59,4)^{(+)}$ & $30(48,4)$ & $14(53,9)$ & $5(15,0)^{(-)}$ & $106(52,0)$ & $0 \Omega$ \\
\hline Cont & & Ocorre & $39(40,6)^{(-)}$ & $32(51,6)$ & $12(46,1)$ & $15(75,0)^{(+)}$ & $98(48,0)$ & 0,040 \\
\hline . & & Não ocorre & $93(96,9)$ & $59(95,2)$ & $23(88,46)$ & $16(80,0)$ & $191(93,6)$ & \\
\hline & & Ocorre & $3(3,1)$ & $3(4,8)$ & $3(11,5)$ & $4(20,0)$ & $13(6,4)$ & \# \\
\hline & $\rightarrow 0$ & Não ocorre & $94(97,9)^{(+)}$ & $57(91,9)$ & $21(80,8)^{(-)}$ & $15(75,0)^{(-)}$ & $187(91,7)$ & 008 \\
\hline Ish & & Ocorre & $2(2,1)^{(-)}$ & $5(8,1)$ & $5(19,2)^{(+)}$ & $5(25,0)^{(+)}$ & $17(8,3)$ & 0,001 \\
\hline & $\rightarrow$ dore & Não ocorre & $96(100,0)$ & $60(96,8)$ & $25(96,2)$ & $17(85,0)$ & $198(97,0)$ & $\#$ \\
\hline & $\rightarrow$ aors & Ocorre & $0(0,0)$ & $2(3,2)$ & $1(3,8)$ & $3(15,0)$ & $6(3,0)$ & \# \\
\hline & $\rightarrow \mathrm{lah}$ & Não ocorre & $96(100,0)$ & $60(96,8)$ & $25(96,2)$ & $16(80,0)$ & $197(96,6)$ & \\
\hline & 7 Lav & Ocorre & $0(0,0)$ & $2(3,2)$ & $1(3,8)$ & $4(20,0)$ & $7(3,4)$ & \# \\
\hline & $\rightarrow$ Ant & Não ocorre & $84(87,5)$ & $47(75,8)$ & $19(73,1)$ & $18(90,0)$ & $168(82,4)$ & 0115 \\
\hline Cor & & Ocorre & $12(12,5)$ & $15(24,2)$ & $7(26,9)$ & $2(10,0)$ & $36(17,6)$ & 0,115 \\
\hline Cor & $\rightarrow$ Ant & Não ocorre & $55(57,3)^{(+)}$ & $34(54,8)$ & $8(30,8)^{(-)}$ & $5(25,0)^{(-)}$ & $102(50,0)$ & \\
\hline & & Ocorre & $41(42,7)^{(-)}$ & $28(45,2)$ & $18(69,2)^{(+)}$ & $15(75,0)^{(+)}$ & $102(50,0)$ & \\
\hline & & Não ocorre & $91(94,8)$ & $58(93,6)$ & $24(92,3)$ & $15(75,0)^{(-)}$ & $188(92,2)$ & \\
\hline & & Ocorre & $5(5,2)$ & $4(6,4)$ & $2(7,7)$ & $5(25,0)^{(+)}$ & $16(7,8)$ & 0,026 \\
\hline & & Não ocorre & $95(99,0)$ & $61(98,4)$ & $26(26,0)$ & $17(85,0)$ & $199(97,6)$ & \\
\hline & $\rightarrow$ Lab & Ocorre & $1(1,0)$ & $1(1,6)$ & $0(0,0)$ & $3(15,0)$ & $5(2,4)$ & \# \\
\hline Dors & & Não ocorre & $86(89,6)^{(+)}$ & $51(82,3)$ & $21(80,8)$ & $10(50,0)^{(-)}$ & $168(82,4)$ & $<0,001$ \\
\hline & & Ocorre & $10(10,4)^{(-)}$ & $11(17,7)$ & $5(19,2)$ & $10(50,0)^{(+)}$ & $36(17,6)$ & $<0,001$ \\
\hline
\end{tabular}

Legenda: DL - Desvio Leve; DML - Desvio Moderado Leve; DMG - Desvio Moderado Grave; DG - Desvio Grave.

Nota: $p$ = significância do teste de Associação Qui-Quadrado;

Análise de resíduos: (+) Associação significante positiva (-) Associação significante negativa.

Figura 5 - Análise das substituições dos traços distintivos conforme a gravidade do desvio fonológico

diferentes gravidades, percorreram as rotas D1 e B2, exceto um classificado como Grave.

As rotas que apresentaram maior frequência de não terem sido percorridas são C4 e B5, seguidas de D3 e C3, que especificam os traços de níveis mais distantes (nível 9 e 8 respectivamente), para todas as gravidades do desvio fonológico. Esse achado corrobora o estudo ${ }^{23}$ que afirma que os traços terminais, convergentes de rotas mais distantes em relação ao Estado 0 , na geometria, são os que mais sofrem alterações. Ainda concorda com outro estudo ${ }^{16} \mathrm{em}$ que as rotas finais são as que mais sofrem alteração, podendo não estar percorrida tanto nos desvios mais leves (Leve e Moderado-Leve) como nos mais graves (Grave e Moderado-Grave), embora no desvio Grave sejam mais frequentemente não percorridas. 
Nos desvios mais leves (Leve o Moderado-Leve), em geral, as rotas não percorridas dizem respeito às rotas finais, enquanto que nos desvios mais graves (Grave e Moderado-Grave), além das rotas finais as rotas iniciais, também apresentam-se, frequentemente, não percorridas, concordando com os dados de outros estudos ${ }^{16,18}$. Além disso, esses achados comprovam haver maior comprometimento nos sistemas fonológicos classificados como Grave e Moderado-Grave de acordo com o Modelo Linguístico Fuzzy20.

Referente à variável nível de complexidade os achados concordam com a literatura ${ }^{15,16}$ em que os sistemas fonológicos mais graves (Grave e Moderado-Grave) atingem nível de complexidade mais baixo. Além disso, os achados estão de acordo com os dados de outros estudos ${ }^{16,18}$ que revelam que os desvios fonológicos mais leves apresentam fonemas de maior nível de complexidade. Corroborando, estudo ${ }^{7}$ refere que o nível de complexidade dos sistemas fonológicos variam consideravelmente, exercendo influência sobre a gravidade do desvio fonológico.

Quanto à variável Aquisição de Fonemas, os achados sustentam a hipótese que gravidade do desvio fonológico acentua-se conforme diminui a aquisição dos fonemas. Esse achado concorda com os dados de outros estudos ${ }^{16,24-26}$ os quais revelam haver correlação entre a gravidade do desvio fonológico e a aquisição dos fonemas ou a quantidade de fonemas alterados (não adquiridos e parcialmente adquiridos). Ainda, tais achados permitem afirmar que nos desvios mais leves há maior número de fonemas adquiridos que nos desvios mais graves estando de acordo com dados de outros estudos ${ }^{16-18,24-26}$. Por fim, os achados corroboram estudo ${ }^{15}$ que afirma que os sons que sofrem mais alterações de produção são aqueles adquiridos mais tarde na sequência do desenvolvimento.

Em relação aos fonemas e a gravidade, no desvio Leve o fonema ausente mais frequente é a líquida não-lateral / $r /$, concordando com outros estudos ${ }^{16,24}$. Ainda, concordam com estudo ${ }^{5}$ em que as alterações mais comuns verificadas nos desvios leves estão relacionadas à aquisição do fonema /r/ e às estruturas silábicas complexas, aquisições características da etapa final da aquisição fonológica.

No desvio Moderado-Leve os fonemas frequentemente ausentes são /r/, /z/, /z/, / $N$ e / $/$ /. Tais achados corroboram o fato de desvios mais leves apresentarem ausentes os fonemas de maior complexidade e de aquisição mais tardia na aquisição normal. Além disso, concordam que os fonemas pertencentes às classes das líquidas e das fricativas são os de aquisição mais tardia ${ }^{1,24,27}$.
No desvio Moderado-Grave os fonemas frequentemente ausente são /r/, /3/, / $/ / / \mathrm{b} /, / \mathrm{g} /, / \mathrm{S} / \mathrm{e}$ /z/. Tais achados revelam que no desvio Moderado-Grave, além de fonemas pertencentes às classes das líquidas e fricativas, que comumente aparecem ausentes nos desvios mais leves, há, na maioria dos casos, comprometimento de fonemas das classes das plosivas, principalmente /b/ e /g/.

No desvio Grave os fonemas mais alterados são /r/, /N/, ///, /R/, /3/, /g/, /J/, /z/, /d/ e /v/. Esses achados mostram, em geral, que todos os fonemas pertencentes à classe das líquidas estão ausentes no desvio Grave. Corroborando, estudo ${ }^{16}$ afirma que na classe das líquidas o fonema de aquisição mais inicial e estável é a líquida não-lateral ///, que mostra-se ausente apenas para desvios fonológicos de maior gravidade.

Ainda, é frequente, no desvio Grave as fricativas /3/, /J/, /z/ e /v/ e as plosivas /g/ e /d/ estarem ausentes. Logo, tais achados apontam que no desvio Grave, comumente, há o comprometimento de fonemas pertencentes às classes das plosivas, fricativas e líquidas, concordando com os dados de outros estudos ${ }^{16,26,28}$. Por fim, quando uma das nasais e/ou das plosivas /t/, /p/ e / $/ \mathrm{k} /$ estiver(m) ausente(s), trata-se de desvio Grave.

Embora a análise das classes de sons não foi adotada no Modelo Linguístico Fuzzy ${ }^{20}$ como variável, os achados evidenciam que o número de classes de sons alteradas mantém relação com a gravidade do desvio fonológico classificada de acordo com o Modelo $^{20}$. Ainda revela que a gravidade acentua-se conforme aumenta o número de classes de sons alteradas, estando de acordo com dados de outro estudo ${ }^{16}$.

A classe se som frequentemente, alterada é a das líquidas. Tal achado concorda com estudos que apontam que as líquidas são as classes de aquisição mais tardia tanto na aquisição normal quanto nos desvios fonológicos ${ }^{1,17,29}$. Ainda, concorda com outros estudos $^{15,16,24}$ que apontam ser a classe das líquidas, frequentemente, as mais alteradas no desvio fonológico.

A segunda classe de sons mais alterada é a classe das fricativas, que se apresentou alterada em $52,9 \%$ dos sistemas fonológicos. Corroborando, outros estudos ${ }^{1,24,27}$ apontam que esta classe, seguida da classe das líquidas, são as de aquisição mais tardia.

As classes das nasais e das plosivas apresentaram-se alteradas com menor frequência 2,0 e $34,8 \%$, respectivamente. Concordando com os dados de estudos ${ }^{16,24}$ que revelam que as classes das nasais e das plosivas estão menos alteradas nos desvios fonológicos. 
Referente à gravidade, verifica-se que no desvio Leve a classe de sons mais alterada é a das líquidas, no desvio Moderado-Leve das líquidas e das fricativas e nos graus Moderado-Grave e Grave das líquidas, das fricativas e das plosivas. Ainda, quando a classe das nasais se apresenta alterada trata-se, em geral, de um desvio Grave.

Quanto aos traços distintivos, que são unidades básicas de caráter acústico e articulatório, na qual estabelecem distinção entre fonemas de um inventário ${ }^{30}$, os achados estão de acordo com os verificados em outros estudos ${ }^{15,16,24,26,28}$, em que desvios mais leves apresentam menor quantidade de substituições de traços distintivos que os desvios mais graves. Tais achados sustentam a hipótese que a análise de traços distintivos é importante para mensurar a gravidade do desvio fonológico e que - Modelo Linguístico Fuzzy ${ }^{30}$ é capaz de diferenciar os graus quanto à quantidade de substituições de traços distintivos.

A ocorrência de diversos tipos de substituições de traços distintivos, verificada nos diferentes graus, concordam com estudos ${ }^{16,23,28}$ que afirmam que as crianças com desvios fonológicos, além de apresentarem dificuldade no uso do traço [voz], também mostram problemas nas distinções de ponto de articulação.

No desvio Leve os traços distintivos mais alterados foram aproximante, vocoide e coronal, para as seguintes substituições: [+aprox] $\rightarrow$ [-aprox], [-voc] $\rightarrow$ [+voc] e [cor +ant] $\rightarrow$ [cor -ant]. Esses achados estão de acordo com os dados verificados em outro estudo ${ }^{24}$. Além disso, as substituições correspondentes aos traços aproximante e vocóide indicam presença de processo de semivocalização de líquida e, a substituição de [cor +ant] $\rightarrow$ [cor -ant] a presença do processo de anteriorização de fricativa.

No desvio Moderado-Leve os traços distintivos mais alterados foram voz e contínuo, para as seguintes substituições: [+voz] $\rightarrow$ [-voz] e [+cont] $\rightarrow$ [-cont]. Ainda, as substituições dos traços aproximante [+aprox] $\rightarrow$ [-aprox], vocoide [-voc] $\rightarrow[+$ voc] e coronal [cor +ant] $\rightarrow$ [cor -ant], também foram frequentes. Esses achados sugerem que além dos processos de semivocalização de líquida e de anteriorização de fricativa, verificados no desvio Leve, ocorrem frequentemente no desvio Moderado-Leve processo de dessonorização e substituição de líquida não lateral por líquida lateral.

No desvio Moderado-Grave os traços distintivos mais alterados foram voz, coronal, aproximante e vocóide, para as seguintes substituições: [+voz] $\rightarrow$ [-voz]; [cor -ant] $\rightarrow$ [cor + ant]; [+aprox] $\rightarrow$ [-aprox] e [-voc] $\rightarrow$ [+voc]. E no desvio Grave os traços distintivos mais alterados foram aproximante, vocóide, contínuo, coronal, voz e dorsal, para as seguintes substituições: [+aprox] $\rightarrow$ [-aprox]; [-voc] $\rightarrow$ [+voc]; [+cont] $\rightarrow$ [-cont]; [cor -ant] $\rightarrow$ [cor +ant]; [+voz] $\rightarrow$ [-voz]; e [dors] $\rightarrow$ [cor].

Corroborando, estudo ${ }^{15}$ também verificou que o desvio grave apresentou maiores médias de ocorrência de alterações dos traços vocóide, contínuo, vozeado, coronal, labial, e dorsal, havendo diferença estatisticamente significante quando comparadas às médias de ocorrência nos graus moderado-severo e leve-moderado.

\section{CONCLUSÃO}

A análise isolada das variáveis utilizadas no Modelo Linguístico Fuzzy ${ }^{20}$ revela que todas mantêm relação com a gravidade do desvio fonológico e que as mesmas são capazes de diferenciar quantitativamente os graus, trazendo informações importantes e coerentes. Quanto ao comportamento dessas variáveis, para Percurso das Rotas a gravidade acentua-se com a diminuição das rotas percorrridas. Além disso, nos desvios mais leves (Leve e Moderado-Leve), em geral, as rotas não percorridas dizem respeito às rotas finais, enquanto que nos desvios mais graves (Grave e Moderado-Grave), além das rotas finais as rotas iniciais, também apresentam-se, frequentemente, não percorridas.

Para Nível de Complexidade o grau Leve alcança, em média, nível de complexidade mais elevado, seguido do grau Moderado-Leve, enquanto que os graus Moderado-Grave e Grave alcança, em média, nível de complexidade mais baixos. Por fim, para Aquisição de Fonemas a gravidade do desvio fonológico acentuou-se conforme diminuiu a aquisição dos fonemas, sendo esta diferença estatisticamente significante. Além disso, observou-se que no grau Leve o fonema, frequentemente, ausente é a líquida não-lateral $/ r /$ enquanto que no desvio Moderado-Leve são os fonemas /r/, /Z/, /z/, / $N$ e /S/. Dessa forma, nos desvios mais leves comumente os fonemas ausentes referem-se aos de maior complexidade e de aquisição mais tardia na aquisição normal.

Ainda, os achados deste estudo permitem concluir que a classificação da gravidade do desvio fonológico por meio do Modelo Linguístico Fuzzy ${ }^{20}$ é capaz diferenciar quantitativamente os graus quanto às classes de sons e aos traços distintivos. 


\section{ABSTRACT}

Purpose: to analyze the behavior of the Path Course, Level of Complexity and acquisition of phonemes, according to the classification of severity of phonological disorders (PD) through the Linguistic Fuzzy Model (BRANCALIONI, 2010). Also, to verify if the classification of severity by means of the Model is able to differentiate quantitatively the degrees or classes of sounds and distinctive features. Method: the sample was made up of the phonological assessment pre-treatment with 204 DF subjects of both genders and aged between four years and two months old to eight years and two months old. All subjects were assessed for phonological evaluation of understanding and expressing language, evaluation of the stomatognathic system, repetitive articulatory examination and audiological assessment. Contrastive and distinctive features analyses were performed. The severity of 204 deviant phonological systems was classified according to the model. The corresponding values of the variables were tabulated and analyzed statistically by Tukey test and Qui-Square Association complemented by analysis of adjusted residuals, considering a significance level of $5 \%(p<0.05)$.

Results: it was verified that the variables of Path Course, Level of Complexity and Acquisition of Phonemes decrease as the severity increases; these relationships are statistically significant. In addition, it was observed that the number of altered sound class and distinctive substitutions sound increase according to the severity; these relationships are statistically significant. Conclusion: the model is able to differentiate the degrees according to the variables, bringing important information according to different degrees.

KEYWORDS: Speech; Speech Disorders; Severity of Illness Index; Classification; Linguistics

\section{REFERÊNCIAS}

1. Lamprecht RR. Aquisição fonológica do Português: perfil de desenvolvimento e subsídios para terapia. Porto Alegre: Artmed; 2004. 232p.

2. Ceron MI, Keske-Soares M, Terapia fonológica: a generalização a itens não utilizados no tratamento (outras palavras). Rev. CEFAC. 2007;9(4):453-60.

3. Wertzner HF, Pagan LO, Galea DES, Papp ACCS. Transtorno fonológico e histórico de otite média. Rev. Soc. Bras. Fonoaudiol. 2007;12(1):41-7.

4. Keske-Soares M, Mota HB, Pagliarin KC, Ceron MI. Estudo sobre os ambientes favoráveis à produção da liquida não lateral $/ r /$ no tratamento do desvio fonológico. Rev. Soc. Bras. Fonoaudiol. 2007;12(1), 48-54.

5. Pagliarin KC, Brancalioni AR, Keske-Soares M, Souza APR. Relação entre gravidade do desvio fonológico e fatores familiares. Rev. CEFAC. 2011;13(3):414-27.

6. Souza APR, Pergher GL, Pagliarin KC. Aspectos motores corporais e orais em um grupo de crianças com transtorno/atraso fonológico. Rev. Soc. Bras. Fonoaudiol. 2010;15(2):226-30.

7. Mota HB. Fonologia: intervenção. In: Ferreira LF, Befi- Lopes DM, Limongi SCO. Tratado de Fonoaudiologia, cap. 63, p.787-814, 2004.

8. Smith SD, Bruce FP, Broada R, Shriberg L. Linkage of Speech Sound Disorder to Reading
Disability Loci. J Child Psychol Psychiatry. 2005;46(10):1057-66.

9. Klein ES, Flint CB. Measurement of Intelligibility in Disordered Speech. Lang Speech Hear Serv Schools. 2006;37:191-9.

10. Donicht G, Pagliarin KC, Mota HB, KeskeSoares M. Julgamento perceptivo da gravidade do desvio fonológico por três grupos distintos. Revista CEFAC. 2010; 12:21-6.

11. Flipsen Jr P, Hummer JB, Yost KM. Measuring severity of involvement in speech delay: segmental and whole-word measures. Am. J. Speech-Lang. Path. 2005;14:298-312.

12. Skahan SM, Watson M, Lof GL. Speechlanguage pathologists' assessment practices for children with suspected speech sound disorders: results of a national survey. Am. J. Speech-Lang. Path. 2007;16:246-59.

13. Williams A. L. Multiple oppositions: theoretical foundations for an alternative contrastive intervention framework. Am. J. Speech-Lang. Path. 2000;9:282-8.

14. Wertzner HF, Amaro L, Teramoto S. Descritores da classificação da gravidade do distúrbio fonológico. Pró-Fono. 2004;16(2):139-50.

15. Pereira LF. Desvio fonológico: desempenho de pré-escolares em tarefas linguísticas e metalinguísticas nos diferentes graus de gravidade. 2006. 292f. Tese (Doutorado em Ciências) 
Universidade Federal de São Paulo - Escola Paulista de Medicina, São Paulo, 2006.

16. Keske-Soares M. Terapia fonoaudiológica fundamentada na hierarquia implicacional dos traços distintivos aplicada em crianças com desvios fonológicos. 2001. 193f. Tese (Doutorado em Letras - Linguística Aplicada), Pontifícia Universidade Católica do Rio Grande do Sul, Porto Alegre, 2001.

17. Lazzarotto-Vulcão C, Matzenauer CLB. A severidade do desvio fonológico com base em traços. Letras de Hoje. 2008;43(3):47-53.

18. Duarte SH. Relação de distância e de complexidade entre traços distintivos na generalização em terapia de desvios fonológicos. 2006. 326f. Dissertação (Mestrado em Letras) Universidade Católica de Pelotas, Pelotas, 2006.

19. Shriberg LD, Austin D, Lewis BA, McSweeny JL, Wilson DL. The percentage of consonants corrects (PCC) metric: extensions and reability data. J Speech Lang Hear Res. 1997;40:708-22.

20. Brancalioni, AR. Proposta de Classificação da Gravidade do Desvio Fonológico por meio da Modelagem Fuzzy segundo o Modelo Implicacional de Complexidade de Traços [dissertação] Santa Maria (RS): Universidade Federal de Santa Maria Mestrado em Distúrbios da Comunicação Humana; 2010.

21. Yavas M, Hernadorena CM, Lamprecht RR. Avaliação fonológica criança: reeducação e terapia. Porto Alegre: Artes Médicas, 2ª Ed. 2002.

22. Shriberg LD. Five subtypes of developmental phonological disorders. Clin. Comm. disorders. 2004;4:38-53.
23. Vaucher AV. Descrição das substituições consonantais presentes nos desvios fonológicos evolutivos: uma abordagem autossegmental. Dissertação. (Mestrado em Letras - Linguística Aplicada) - Pontifícia Universidade Católica do Rio Grande do Sul, Porto Alegre, 1996.

24. Casarin M. Prevalência dos desvios de fala em pré-escolares de escolas públicas estaduais de Santa Maria-RS. 2006. 116f. Dissertação (Mestrado em Distúrbios da Comunicação Humana) Universidade Federal de Santa Maria, Santa Maria, 2006.

25. Keske-Soares M, Brancalioni AR, Marini C, Pagliarin KC, Ceron MI. Eficácia da terapia para desvios fonológicos com diferentes modelos terapêuticos. Pró-Fono. 2008;20(3):153-8.

26. Pagliarin KC, Mota HB, Keske-Soares M. Análise da eficácia terapêutica em três modelos fonológicos de abordagem contrastiva. Pró-Fono. 2009;21(4):297-302.

27. Pagan LO, Wertzner HF. Análise acústica das consoantes líquidas do Português Brasileiro em crianças com e sem transtorno fonológico. Rev. Soc. Bras. Fonoaudiol. 2007;12(2):106-13.

28. Ceron MI, Keske-Soares M, Gonçalves GF. Escolha dos sons-alvo para terapia: análise com enfoque em traços distintivos. R. Soc. Bras. Fonoaudiol. 2010;15(2): 270-6.

29. Cruz GF. O processo de semivocalização de líquidas laterais em posição pré-vocálica: uma revisão teórica. Letrônica. 2009;2(2):48-58.

30. Matzenauer CL. A generalização em desvios fonológicos: o caminho pela recorrência de traços. Letras de Hoje. 2008;43(3):27-34.

Endereço para correspondência:

Ana Rita Brancalioni

Rua Guerino Catapan, 342

Ibiraiaras - RS

CEP: 95305-000

E-mail: fonoaninha@yahoo.com.br 